\title{
Propuesta de Instrumento para evaluar la producción científica de las universidades en Centroamérica ${ }^{1}$.
}

\section{Porposal of an Instrument to evaluate the scientific production of the universities in Central America.}

\author{
Karla Rodríguez-Burgos ${ }^{2}$ \\ Cristian Rivas-Castillo ${ }^{3}$ \\ RESUMEN
}

El presente artículo tiene como objetivo diseñar un instrumento para medir la producción científica de las universidades en Centroamérica. El contenido del instrumento se diseñó a través de la metodología propuesta por Orantes Pineda (2015), CONACYT (2011), Castro Rodríguez (2017), Rueda-Barrios \& Rodenes-Adam (2016), así como Sáenz López \& Rodríguez-Burgos (2014), así como de la experiencia de los autores. A partir de un análisis de contenido de estudios previos se diseñó un instrumento cuantitativo denominado Modelo de Evaluación Científica Rodríguez-Burgos \& Rivas-Castillo, clasificado desde la teoría y la práctica en dos conceptos, 4 dimensiones, 21 indicadores y conformado por 120 ítems que evalúan el proceso de formación investigadora que reciben los docentes que realizan investigación en las universidades. Por otro lado también mide las habilidades adquiridas y como esto influye en la producción científica de ellos y sus instituciones, el instrumento se divide en dos conceptos procesos de formación y desarrollo de habilidades, los cuales abarcan cuatro dimensiones: capacitación en proyectos, capacitación en publicación y divulgación científica, realización de proyectos y visibilidad científica. La aplicación de este instrumento en Centroamérica permitirá determinar las áreas de oportunidad que tienen los países para aparecer en los rankings internacionales que evalúan la calidad internacional de las universidades.

\footnotetext{
1El presente artículo es resultado parcial de investigación de la tesis de grado de Maestría en Relaciones Internacionales, Facultad de Ciencias Políticas y Relaciones Internacionales en la Universidad Autónoma de Nuevo León titulada "Nivel de producción científica en las Universidades de Nicaragua" financiado por el CONACyT en México.

2 Profesora e Investigadora de tiempo completo. Especialista en aplicación y análisis de métodos cualitativos y cuantitativos. Cuenta con perfil PRODEP de la Secretaría de Educación y en miembro del Sistema Nacional de Investigadores del CONACyT. Facultad de Ciencias Políticas y Relaciones Internacionales. Universidad Autónoma de Nuevo León. PhD en Filosofía con Acentuación en Ciencias Políticas. karoburgos@yahoo.com.mx. ORCID: 0000-0003-2093-8146

3 Lic. En Derecho por la Universidad Nacional Autónoma de Nicaragua, UNAN-Managua, Maestrando en Relaciones Internacional por la Universidad Autónoma de Nuevo León, UANL. ORCID ID: 0000-0001-6827-1462. Castillocris25@yahoo.com

Recibido: 10 de Agosto de 2018

Aprobado: 22 de Noviembre de 2018.
}

Revista Legem, Universidad del Atlántico 4 (2), Julio - Diciembre 2018, pp. 1-20 ISSN: 2346-2787 


\title{
Palabras claves:
}

Instrumento de evaluación, producción científica, rankings internacionales, formación científica, habilidades investigativa.

\begin{abstract}
The main objective of this article is to design an instrument to measure the scientific production of universities in Central America. The content of the instrument was designed through the methodology proposed by Orantes Pineda (2015), CONACYT (2011), Castro Rodríguez (2017), Rueda-Barrios \& Rodenes-Adam (2016), as well as Sáenz López \& Rodríguez-Burgos (2014), as well as the own experience of the authors. Based on a content analysis of previous studies, the quantitative instrument called the Scientific Evaluation Model Rodríguez-Burgos \& Rivas-Castillo was designed, classified from the theory and practice in two concepts, 4 dimensions, 21 indicators and made up of 120 items that evaluate the process of research training received by teachers who do research in universities. On the other hand, it also measures the skills acquired and as this influences the scientific production of them and their institutions, the instrument is divided into two concepts: training processes and skills development, which encompass four dimensions: project training, publication training and scientific dissemination, project realization and scientific visibility. The application of this instrument in Central America will allow determining the areas of opportunity that countries have to appear in the international rankings that evaluate the international quality of universities.
\end{abstract}

\section{Keywords:}

Evaluation instrument, Scientific production, international rankings, scientific training, investigative skills. 


\section{Introducción}

La investigación científica de acuerdo a Machado Cabezas (2015) es necesaria para el desarrollo de una sociedad, permitiendo aumentar el conocimiento de la comunidad en general, en este sentido Monje Álvarez (2011) expresa que la investigación el instrumento que permite "conocer, explicar, interpretar y transformar la realidad". El mundo actual, reconoce que para su desarrollo es necesario tener como base la investigación y producción científica.

Es así las universidades deben generar investigación científica, ya que su misión no es solo la formación de profesionistas, sino también se debe impulsar la investigación y la difusión de la cultura (Ortega y Gasset, 1930), ya que de acuerdo a Rivera García, Espinoza Manfugas \& Valdez Bencomo (2017) los institutos de educación superior son los encargados de generar investigación y producción científica al tener a los profesores quienes son profesionales experimentados que aportan a los alumnos a través del conocimiento desarrollado y la experiencia adquirida, a quienes buscan impulsar no solo para su formación profesional, sino también para apoyar el progreso de la nación.

Para Duarte de Krummel (2015) la función de investigación que realizan las universidades es una pieza clave de las actividades universitarias. De esta forma, la generación de nuevos conocimientos mediante la investigación y la innovación conforman la infraestructura productiva social y científico-tecnológica de las instituciones de educación superior, lo cual se traduce en aportaciones al desarrollo humano, social y económico de la sociedad. 


\section{Planteamiento del problema}

En los últimos años ha surgido el interés por evaluar la calidad de las universidades tanto de la formación como de la investigación que se desarrolla en ellas, además se ha hecho imperiosa la necesidad por buscar instrumentos que analicen el nivel educativo y producción científica global, la cual sirve medir, comparar y contrastar los centros universitarios entre sí, surgiendo institutos que por medio de rankings miden la función investigativa de las Instituciones de Educación Superior (IES).

Los rankings universitarios tienen como objetivo jerarquizar las instituciones de educación superior, basándose en indicadores que permiten la medición de "la calidad de la educación universitaria, de la investigación y otros aspectos de la actividad académica" Tomas Folch, Feixas, Bernabéu-Tamayo \& Ruiz Ruiz (2015).

Según López Leiva (2012) entre los indicadores que se utilizan para medir la función investigativa de las universidades se encuentran los fondos de investigación obtenidos, el número de publicaciones que las universidades realizan, el número de citas que las publicaciones reciben, por los premios y reconocimientos otorgados a los profesores de la institución y por el número de patentes que realizan.

Es a partir de la publicación de los rankings que Córdoba González, Marín Campos, Polanco Cortez \& Villegas Rojas (2012) notan una problemática de escasez en la investigación que se realiza en los países centroamericanos al observar que las Instituciones de Educación Superior de esos países tienen poca presencia, siendo que en la clasificación correspondiente al 2017 elaboradas por el World University Rankings (QS), Rankings Web de Universidades (WEBOMETRICS), SCIMAGO Institutions Rankings (SIR), las universidades de esta región, muestran una debilidad en su producción científica.

Por ejemplo en el Ranking QS, el cual para la elaboración de sus clasificaciones utiliza cinco indicadores básicos: impacto de investigaciones y productividad, compromiso con la enseñanza, empleabilidad, impacto en línea e internacionalización, 
se analizaron en la región latinoamericana un total de 385 instituciones de educación superior, dentro de las cuales solo la Universidad Nacional Costa Rica se posicionó entre las 50 mejores instituciones de la región, seguido de la Universidad Tecnológica de Panamá en la posición 101.

Por otra parte, en el Webometrics que tiene como principales indicadores según Isidro Aguillo \& Begoña Granadino (2006) “el número de citas que reciben los autores, así como la cantidad de artículos académicos que los miembros de las universidades publican en revistas internacionales de alto impacto", en el informe correspondiente al año 2018 se evaluaron un total de 3,696 universidades latinoamericanas, siendo la Universidad Nacional de Costa Rica la mejor situada por parte de la región centroamericana ocupando el puesto número 38.

El Scimago Institutions Rankings es otro de las herramientas para evaluar la función de investigación de las universidades de todo el mundo, clasifica a las instituciones académicas que realizan investigación, sus indicadores son la investigación, innovación e impacto social, y son seleccionadas aquellas que cuenten con más de 100 artículos publicados e incluidos en la base de datos Scopus en intervalos de cinco años, en el informe correspondiente al año 2018 se estudiaron un total de 355 instituciones de la región latinoamericana de las cuales ninguna se encuentra en el istmo centroamericano.

Por tanto, podemos indicar que el problema de investigación radica en la escasa posición que presentan las Instituciones de Educación Superior centroamericanas en los rankings internacionales que evalúan la calidad de las universidades latinoamericanas. Es así que el objetivo de esta investigación es diseñar un instrumento de medición que permita evaluar la formación científica de los investigadores, así como el desarrollo de habilidades que tienen a partir de la experiencia.

La importancia de este instrumento se centra en que una vez desarrollado, este puede ser aplicado en las Universidades de Centroamérica permitiendo tener desde 
dentro de cada una de las IES una visión de las áreas de oportunidad que se presentan, proporcionando información relevante para la mejora, en capacitación y desarrollo de habilidades de los investigadores necesarias para el desarrollo de investigaciones o proyectos que incrementen la cantidad de artículos publicados por los docentes investigadores de las instituciones de educación superior y que precisamente generen visibilidad a través de la aparición en los rankings internacionales.

\section{Metodología utilizada}

Para el diseño del instrumento se realizó un análisis de contenido, el cual según Berelson (1992) es "una técnica de investigación para la descripción objetiva, sistemática y cuantitativa del contenido manifiesto de la comunicación", mientras que para Krippendorff (1990) es una técnica que permite realizar inferencias a partir de ciertos datos, las cuales se pueden reproducir y validar para aplicarse a su contexto. Esta técnica es aplicable para el estudio de documentos escritos.

La misión de esta técnica es la de "recopilar, comparar y clasificar expresiones para relacionarlas con el sistema expresivo al que pertenecen" (Navarro \& Díaz, 1995), permitiendo conocer acerca de la fuente de comunicación, los autores, receptores y el contexto en el que se lleva a cabo (López Barajas, 1991). Es por tanto que la transformación de la literatura la hace el investigador a partir de reglas definidas con anterioridad, y teóricamente justificadas, con una interpretación adecuada de acuerdo a Cámara Estrella (2009) y finalmente los resultados obtenidos deben ser verificados y sistematizados que permitan observar todo el contenido Andreu (1998).

Se inició entonces con una búsqueda bibliométrica con el objetivo de identificar investigaciones relacionadas con el proceso de formación y desarrollo de habilidades en la investigación aplicadas con anterioridad, esto como punto de partida para 
realizar el proceso de construcción del cuestionario. Además, se tuvo en cuenta la opinión de expertos que han realizado investigaciones o proyectos de investigación.

Como parte de la literatura analizada, se localizaron instrumentos e indicadores que aportaron a la construcción del cuestionario, los cuales fueron utilizados en investigaciones previas, como la tesis doctoral "Diagnostico de la producción Científica y actividad investigadora del profesorado de las instituciones de educación superior en El Salvador" Orantes Pineda, (2015), así como del Consejo Nacional de Ciencia y Tecnología de El Salvador (CONACYT, 2011).

\section{Justificación del instrumento.}

Este instrumento se generó producto de la consulta bibliométrica que se realizó, donde se descubrió la importancia que otros autores le han dado al estudio de la formación y desarrollo de habilidades con las que deben de contar los investigadores en las universidades para la generación de producción científica, el modelo que se expresa a continuación esta modificado de los modelos propuestos por Orantes Pineda (2015), CONACYT de El Salvador (2011), Castro Rodríguez (2017), RuedaBarrios \& Rodenes-Adam (2016), Sáenz López \& Rodríguez-Burgos (2014) debido a que se pudo inferir que lo propuesto por los autores se limitaba al estudio de determinadas variables en relación a la formación y habilidades que los investigadores debían tener.

El cuestionario elaborado retoma elementos como ítems e indicadores propuestos en estos documentos, con el objetivo de formular un instrumento que evalué todos los componentes que debe de poseer el investigador universitario para el cumplimiento de todas las etapas de la investigación.

Es así que se diseñó el instrumento de medición, el cual permitirá evaluar la formación investigadora, actividad investigativa y producción científica de los docentes-investigadores de las universidades, además de conocer la realidad de las 
universidades en relación a la función de investigación que estas desarrollan, así como el grado de conocimiento que tienen sus investigadores.

\section{Construcción del instrumento}

Para la construcción del instrumento se sigue la estructura propuesta por Cea D Ancona (2001) la cual permite generar un concepto general del tema, la dimensión que se quiere evaluar, más específicamente los indicadores o variables empíricas por medir para finalmente tener lo ítems.

Es así que para Cea D Ancona (2001) los conceptos cumplen una función de síntesis clasificando los objetos, mientras que la dimensión es la separación de conceptos sumamente complejos, para cada dimensión de un concepto es un aspecto relevante que, en conjunto, resumen o integran el concepto teórico, esta es una propiedad del concepto la cual no se podría observar empíricamente, por tanto, las dimensiones representan aproximaciones al concepto que miden.

Además de lo anterior, los indicadores se encuentran relacionadas con propiedades latentes o no observables (dimensión), finalmente se generan los ítems, los cuales permiten medir a partir de determinadas cuestiones a los indicadores. Por lo tanto, los conceptos, dimensiones e indicadores se elaboraron haciendo uso de la literatura encontrada en la cual se propusieron instrumentos para evaluar a los investigadores, de estos modelos se han excluidos algunos valores y redefinidos otros, en función del objetivo de nuestra investigación.

Por lo tanto, a partir de la literatura encontrada tanto metodológica como científica se hace la propuesta de diseñar un instrumento, el cual denominaremos Modelo de Evaluación de Producción Científica Rodríguez-Burgos \& Rivas-Castillo, el cual se presenta en la tabla 1. 
Tabla 1. Modelo de Evaluación de Producción Científica Rodríguez-Burgos \& RivasCastillo

\begin{tabular}{|c|c|c|c|}
\hline $\begin{array}{l}\text { CLASIFICA } \\
\text { CIÓN }\end{array}$ & $\begin{array}{l}\text { CONCEPTO } \\
\mathrm{S}\end{array}$ & DIMENSIONES & $\begin{array}{c}\text { INDICADORES } \\
\text { (VARIABLE EMPÍRICA) }\end{array}$ \\
\hline \multirow{5}{*}{ 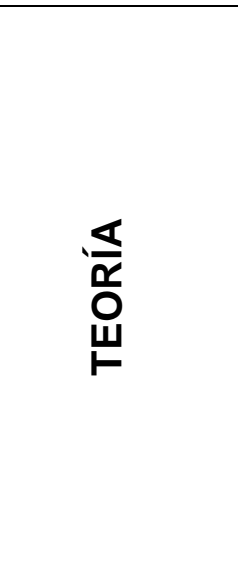 } & \multirow{5}{*}{$\begin{array}{l}\text { PROCESO } \\
\text { DE } \\
\text { FORMACIÓ } \\
\mathbf{N}\end{array}$} & \multirow{3}{*}{$\begin{array}{l}\text { CAPACITACIÓN EN } \\
\text { PROYECTOS }\end{array}$} & Estructura metodológica \\
\hline & & & Aplicación metodológica \\
\hline & & & $\begin{array}{l}\text { Análisis y reporte de } \\
\text { resultados }\end{array}$ \\
\hline & & \multirow{2}{*}{$\begin{array}{l}\text { CAPACITACIÓN EN } \\
\text { PUBLICACIÓN Y } \\
\text { DIVULGACIÓN } \\
\text { CIENTÍFICA }\end{array}$} & Publicación \\
\hline & & & Divulgación \\
\hline \multirow{14}{*}{ 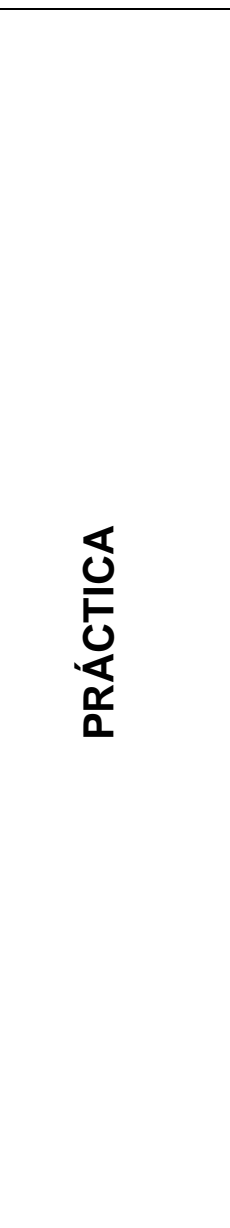 } & \multirow{14}{*}{$\begin{array}{l}\text { DESARROL } \\
\text { LO DE } \\
\text { HABILIDAD } \\
\text { ES }\end{array}$} & \multirow{6}{*}{$\begin{array}{l}\text { REALIZACIÓN DE } \\
\text { PROYECTOS }\end{array}$} & Habilidades de estructura \\
\hline & & & Habilidades de aplicación \\
\hline & & & Habilidades de análisis \\
\hline & & & Habilidades de escritura \\
\hline & & & $\begin{array}{l}\text { Habilidades de } \\
\text { negociación }\end{array}$ \\
\hline & & & Apoyo universitario \\
\hline & & \multirow{8}{*}{ VISIBILIDAD CIENTÍFICA } & Publicaciones en artículos \\
\hline & & & $\begin{array}{l}\text { Publicaciones en } \\
\text { capítulos libros }\end{array}$ \\
\hline & & & Publicaciones en artículos \\
\hline & & & $\begin{array}{l}\text { Publicaciones } \\
\text { interinstitucionales }\end{array}$ \\
\hline & & & Indicadores de visibilidad \\
\hline & & & Normas éticas \\
\hline & & & Tesis de grado \\
\hline & & & Divulgación en redes \\
\hline
\end{tabular}




\begin{tabular}{|l|l|l|}
\hline & & Divulgación científica \\
\cline { 3 - 3 } & & Redes de investigación \\
\hline
\end{tabular}

Elaboración propia

El modelo propuesto en la tabla 1 se puede clasificar inicialmente en dos partes, la teoría y la práctica. Desde la parte teórica se puede observar como concepto el proceso de formación el cual incluye la capacitación que han tenido los investigadores para la realización de proyectos, así como la capacitación para que una vez desarrollado el proyecto se generen publicaciones científicas y la forma en la que serán divulgadas.

Por otro lado, en la segunda dimensión, la cual clasificamos como la práctica, se engloban las habilidades que desarrollan los investigadores cuando llevan a cabo proyectos de investigación, a la vez que también se quiere observar la visibilidad científica que desarrollan para promover sus investigaciones.

En el siguiente apartado se describe de manera puntual los conceptos del proceso de formación y el desarrollo de habilidades, así como las dimensiones que lo componen, los indicadores a los que hacen referencia y al final una visión general del contenido de los ítems.

\section{Descripción de la propuesta del Modelo de Evaluación de Producción Científica}

A continuación, se desarrollará el análisis de los elementos que integran el cuestionario, resaltando la finalidad de cada concepto, dimensión e indicador que se propone.

El concepto de proceso de formación, involucra el aprendizaje, la adquisición de conocimientos, habilidades y capacidades que todo investigador debe de poseer para la realización de una investigación, es decir se pretende englobar todas las competencias con las que se le debe de dotar al investigador. Este concepto se 
dividió en dos dimensiones, capacitación en proyectos, así como la capacitación en publicación y divulgación científica, entendiendo a la capacitación de proyectos como el conjunto de actividades didácticas orientadas a cumplir las necesidades de ampliación de conocimientos en la elaboración de proyectos de investigación.

Como parte de la CAPACITACIÓN EN PROYECTOS se evaluará los conocimientos adquiridos en la elaboración de estructura metodológica. En este apartado se tomará en cuenta la formación que se tiene como investigador, así como aquellos elementos básicos en toda investigación como el uso correcto de base de datos, la elaboración de matriz de congruencia, la estructuración de proyectos de investigación, la formulación de hipótesis, así como la comprobación de las mismas. Este apartado está compuesto de 11 ítems.

También se evaluará la aplicación metodológica en donde se realizaron 5 ítems preguntando a los investigadores si se les ha capacitado en la selección de los métodos de aplicación tanto cualitativa como cuantitativa, la habilidad que tienen o han adquirido en el diseño de instrumentos de medición y los pasos que deben de seguir para el desarrollo de esta etapa como la selección de la muestra y el pilotaje del instrumento diseñado.

Como último indicador de la dimensión de capacitación de proyectos se tiene el análisis y reporte de resultados, los cuales se miden a través de 6 ítems, donde algunos de ellos fueron tomados del modelo propuesto por Orantes Pineda (2015), y pretende conocer la competencia que tienen los investigadores en el manejo de software especializado para capturar y analizar tanto información cualitativa como cuantitativa, así como las bases de datos donde estos realizan la búsqueda de información para sustentar sus proyectos.

\section{La dimensión CAPACITACIÓN EN PUBLICACIÓN Y DIVULGACIÓN CIENTÍFICA} comprende la generación de conocimiento que el investigador debe tener para realizar una correcta publicación y divulgación científica. La publicación se entiende como el hecho de hacer público lo que se hace, permitiendo mostrar a la sociedad 
científica los resultados de una investigación para que el mismo pueda ser consultado, revisado y debatido, siendo este apartado medido a través de 8 ítems.

Con el indicador publicación se evaluará si los investigadores han recibido capacitación para la elaboración de un artículo científico, el conocimiento que estos tienen sobre que publicar y donde publicar ya sean revistas nacionales o extranjeras, así como el acercamiento que han tenido con editores y editoriales de prestigios tanto de su país como externos.

Por otro lado, la divulgación, medida en este instrumento en 6 ítems, es la parte final de la investigación, esto es, la acción y efecto de difundir el conocimiento científico de modo que resulte accesible para el público en general y/o especializado. La parte de divulgación permitirá conocer si los docentes-investigadores registran su nombre científico, la frecuencia con la que suben información a repositorios institucionales y buscadores académicos, y si esta información que suben les genera citas e índices $\mathrm{H}$, asimismo si estos asisten a congresos científicos.

Pasando al segundo concepto de Desarrollo de habilidades se refiere al conjunto de acciones que realiza el investigador para llevar a cabo diferentes actividades, utilizando los conocimientos que posee, es decir, se evaluara si el docente investigador hace uso del conocimiento adquiridos en la etapa de formación investigadora, las dimensiones de este concepto son la realización de proyectos y visibilidad científica.

La REALIZACIÓN DE PROYECTOS se basa en las habilidades que el investigador ha desarrollado para la elaboración de proyectos de investigación, estas habilidades son de estructura, aplicación, análisis, escritura, de negociación y el apoyo que el investigador recibe tanto de las instituciones como de la cooperación extranjera o nacional.

Las habilidades de estructura, a partir de la evaluación en 15 ítems, permitirá conocer si los investigadores realizan o han realizado proyectos de investigación, si son 
capaces de identificar problemas que pueden ser objeto de investigación, si definen variables de estudio, conocer sus fuentes de información, ya sea de base de datos o artículos de revistas y libros, así como también se pretende conocer si esta información les permite sustentar las variables de estudio.

Los 7 ítems relacionados a la aplicación de las habilidades abordarán si han realizado instrumentos de medición cuantitativa y cualitativa, si estos instrumentos han sido piloteados, si han aplicado métodos de muestreo y si estos investigadores utilizan una metodología adecuada para la elaboración de proyectos de investigación.

Las habilidades de análisis, medida en 7 ítems, abordan el uso de codificaciones para el análisis cualitativo, también si los investigadores realizan sus observaciones de acuerdo a software especializados de tipo cuantitativo y/o cualitativo, y si una vez analizados los datos realizan la interpretación de los mismos. Además, si elaboran reportes técnicos y si cuentan con las habilidades para realizar la publicación de los resultados obtenidos del análisis de los datos.

El indicador habilidades de escritura, compuesto por 5 preguntas, permite observar las acciones que los investigadores deben de realizar como redactar artículos científicos con el objetivo que sean publicados tanto en revistas nacionales como extranjeras, así como si realizan citas apegados a las normas internacionales de citación.

En las 7 preguntas de habilidades de negociación se quiere conocer los elementos económicos con los que cuentan los investigadores para la realización de los proyectos de investigación, algunos de los ítems propuestos en este indicador se tomaron de CONACYT de El Salvador (2011), se agregaron ítems para conocer si los investigadores han realizado proyectos sin contar con financiamiento, así como si el personal con el que cuentan es suficiente.

El apoyo universitario es trascendental para conocer el quehacer de los docentes investigadores es por tal motivo que se desarrollan 10 preguntas relacionadas a si la 
universidad o centro de investigación aporta al crecimiento tanto personal como profesional de los docentes y si esta les ofrece incentivos económicos por los resultados que estos obtengan en producción científica, además si la universidad fomenta la alianza con otras universidades o empresas.

Como ultima dimensión del instrumento se desarrolló la VISIBILIDAD CIENTÍFICA la cual permite conocer la realidad de la producción científica de los docentes investigadores, en donde las preguntas están relacionadas a conocer donde están publicando, así como si se fomentan redes de investigación con otras instituciones nacionales o extranjeras. Ciertos ítems de estos indicadores fueron estudiados por Rueda-Barrios \& Rodenes-Adam (2016).

El primer indicador de la dimensión visibilidad científica, es la publicación de artículos que realizan los investigadores, con 9 ítems se pretende medir la cantidad de documentos publicados en revistas universitarias como externas a la institución o bien si han publicado en revistas extranjeras que generen un alto impacto a sus investigaciones.

La publicación en capítulos de libros, de la literatura revisada para la elaboración de este instrumento se encontró que ningunos de los autores que han abordado la problemático han tomado en cuenta la cantidad de publicaciones que un investigador realiza en libros, en este instrumento se desarrolla la importancia que tiene este tipo de publicación para la producción científica, por lo que se agregaron 4 preguntas al cuestionario relacionadas con este punto.

Otro indicador de la visibilidad científica se denominó como publicaciones interinstitucionales, con 3 ítems, preguntando si han realizado proyectos con investigadores de lammisma universidad, así como investigadores de otras universidades nacionales y extranjeras.

Los 12 ítems que representan los indicadores de visibilidad, están orientados a conocer si los investigadores tienen dominio del idioma ingles $u$ otro idioma, si las 
publicaciones que han realizado han sido citados, así como se desea conocer si suben sus publicaciones a bases de datos como repositorios institucionales, Scopus, Google Scholar, Academia.edu, Research.edu., entre otros.

El indicador tesis de grado evalúa en 7 ítems si los docentes realizan el rol de tutor de tesis sean estas de pregrado o posgrado, nacionales o internacionales. También, se pretende medir en 3 ítems, por medio del indicador normas éticas si el investigador docente se apega a la ética que todo investigador debe de tener al momento de realizar una investigación y si estos respetan los derechos de los autores de otras investigaciones citándolos correctamente.

La divulgación en redes, en 8 ítems, pretende conocer la forma en la que los investigadores difunden sus trabajos investigativos, si los comparten con sus estudiantes, si utilizan blogs o cuentas de Facebook, twitter o canal de YouTube, programas de radio, programas de televisión o la prensa escrita para dar a conocer a la sociedad su quehacer dentro de la investigación científica.

En el cuestionario también se evalúa el apoyo que la universidad brinda a los investigadores, por medio del indicador divulgación científica, se desarrollan 9 ítems que permitirán conocer si la universidad promueve eventos científicos o bien si esta facilita apoyo económico para que los investigadores acudan a congresos con la finalidad de comunicar los resultados de las investigaciones o proyectos que realizan.

El último indicador de este instrumento se denominó redes de investigación y permitirá por medio de 3 preguntas conocer como los investigadores se relacionan con otros grupos de investigación, sean de su misma universidad u otras universidades nacionales o extranjeras, así mismo se les preguntara si pertenecen a alguna red de investigadores.

\section{Resultados}


A partir de la obtención de los instrumentos aplicados por otros investigadores, así como de la experiencia propia de los investigadores se diseñó un Modelo de Evaluación de Producción Científica, el cual pretende medir desde la parte teórica (si se recibió capacitación), hasta la parte práctica (si se han desarrollado habilidades desde la empiria), el cual consta de 120 ítems clasificados en 4 dimensiones y 21 indicadores, el cual se pretende, una vez realizadas las validaciones interna y externa correspondientes (ranking de expertos, pilotajes y Alpha de Cronbach) permitirá observar desde el punto de vista de los investigadores las áreas de oportunidad que se presentan en las universidades en los países centroamericanos.

El cuestionario tiene validez de contenido, lo cual constituye un importante indicador de su valor, este instrumento una vez publicado pasara a la fase de pilotaje por lo que podría modificar su contenido y posterior se aplicara para obtener resultados de la producción científica de las universidades centroamericanas.

En relación con su formación como investigadores se diseñaron ítems para evaluar las capacitaciones en investigación que han recibido, sean técnicas de investigación o el manejo de las herramientas de investigación, mientras que en la dimensión de desarrollo de habilidades los ítems estudian la cantidad de artículos que los docentes investigadores han realizados y si estos han culminado el proceso investigativo con la publicación, divulgación y participación en redes de investigación.

\section{Conclusiones}

El instrumento diseñado constituye una herramienta importante en la evaluación de la producción científica de los docentes investigadores de las universidades, ya que permitirá conocer el nivel de formación investigadora de estos y como aplican los 
conocimientos adquiridos en la etapa de capacitación para lograr una significativa producción científica.

Esta propuesta de modelo permitirá encaminar futuras investigaciones en el tema de la evaluación de la producción científica de las universidades o incluso de un Estado, además se espera con los resultados que se obtengan de su aplicación se podrán trazar políticas y estrategias en relación a la inversión por parte de los países o universidades en investigación, logrando un desarrollo social de sus naciones.

El propósito del modelo al tener una clasificación en dos partes, la teoría de la práctica, tiene que ver con que de acuerdo a la experiencia que se tiene, las Universidades que ya cuentan con un prestigio, dan por hecho que los investigadores ya fueron formados en investigación y se exigen resultados de los mismos, esto es, en muchas ocasiones se saltan los procesos de formación para los investigadores y solo se pide realizar proyectos, publicar en revistas científicas del más alto nivel sin tomar en consideración la capacitación del investigador y solo se les conmina a presentar resultados (directamente la práctica).

Cabe señalar que aunque pareciera que en las Universidades de Centroamérica no se presentan resultados favorables en los rankings, de acuerdo a entrevistas previas con investigadores de algunos países centroamericanos, ellos cuentan con recursos internacionales para llevar a cabo proyectos financiados por organismos internacionales, sin embargo desconocen la forma de producir publicaciones científicas y la manera de divulgarlos, por lo que tienen fallas en la comunicación de la ciencia para generar el impacto que permita que sus universidades puedan figurar en los rankings internacionales.

\section{Referencias.}

Andreu Abela, Jaime. (1998). Las técnicas de Análisis de Contenido: Una revisión actualizada. 
Berelson, B. (1952). Content Analysis in Communication Research, Free Press, Glencoe.

Cámara Estrella, África María. (2009). Construcción de un instrumento de categorías para analizar valores en documentos escritos. REIFOP. 12 (2). 59-72. (Enlace web http://www.aufop.com)

Castro Rodríguez, Y, A. (2017). Factores de influencia y su relación con la producción científica de estudiantes de la facultad de odontología de la universidad nacional mayor de san marco en el 2017. Lima. Perú.

Cea D'Ancona, M. A. (2001). Metodología cuantitativa. Estrategias y técnicas de investigación social. Madrid. Síntesis Sociológica.

CONACYT. (2011). Indicadores de Ciencia y Tecnología 2010. Estadísticas sobre actividades científicas y tecnológicas. Sector de Educación Superior. San Salvador.

Córdoba González, S., Marín campos, A., Polanco Cortes, J., \& Villegas Rojas, M. (2012). La producción Científica indexada de América Central y República Dominicana. Universidad de Costa Ricas, 1-19.

Duarte de Krummel, M. (2015). Importance of scientific research university life. Revista Científica de la UCSA, 1-3.

Isidro, F. Aguillo \& Begoña Granadino. (2006). Indicadores web para medir la presencia de las universidades. Revista de Universidad y Sociedad del Conocimiento.

Krippendorff, K. (1990). Metodología del análisis de contenido. Teoría y Práctica. PAIDOS. comunicación.

López Barajas, E. (1991). Fundamentos de metodología Científica. Madrid: UNED,93. 
López Leiva, S. (2012). Los rankings universitarios. Bases teóricas, Metodología y su Impacto en la Educación Superior Global. Revista De La Educación Superior, 141-149.

Machado Cabezas, A. (2015). Investigación Científica y Utilidad Social: Un Análisis de la Administración de empresas. Encuentros, 1-6.

Monje Álvarez, C.A. (2011). Metodología de la investigación cuantitativa y cualitativa: Guía Didáctica. Neiva.

Navarro, Pablo, \& Díaz, Capitolina. "Análisis de Contenido", En: Delgado, Juan Manuel \& Gutiérrez, Juan: Métodos y Técnicas Cualitativas de investigación en ciencias sociales, Capitulo 7, pp.177-224. Madrid, España.

Orantes Pineda, B. (2015) Diagnostico de la producción científica y actividad investigadora del profesorado de las instituciones de educación superior en El Salvador. Granada, España.

Ortega y Gasset, J. (1930). Misión de la Universidad. Buenos Aires.

Quacquarelli Symonds (QS). (diciembre de 2018). QS World University Rankings. Obtenido de https://www.topuniversities.com/university-rankings/latin-americanuniversity-ranking/2018

RANKING WEB DE UNIVERSIDADES. (2018). RANKING WEB DE UNIVERSIDADES. Obtenido de http://www.webometrics.info/es.

Rivera García, C. G., Espinoza Manfugas, J. M., \& Valdez Bencomo, Y, D. (2017). La investigación científica en las universidades ecuatorianas. Prioridad del sistema educativo vigente. Revista Cubana de Educación Superior.

Rueda-Barrios, G, \& Rodenes-Adam, M. (2016). Factores determinantes en la producción científica de los grupos de investigación en Colombia. Revista Española de Documentación Científica. 
Sáenz López, K., Rodríguez-Burgos, K. (2014). Habilidades investigativas. En Métodos y técnicas cualitativas y cuantitativas aplicables a la investigación en ciencias sociales. EDITORIAL TIRANT HUMANIDADES. pp. 86-95.

Scimago LAB. (DICIEMBRE de 2018). SCIMAGO INSTITUTIONS RANKINGS. Obtenido de https://www.scimagooir.com/rankings.php

Tomas Folch, M., Feixas, M., Bernabéu-Tamayo, M.D., \&Ruiz Ruiz, J.M. (2015). La literatura Científica sobre rankings universitarios: una revisión sistemática. REDU, 33-54. 